\title{
ESTUDO CINÉTICO DA TRANSFORMAÇÃO MARTENSÍTICA NO AÇO MICROLIGADO USISAR 80T
}

\author{
Samuel da Silva de Souza \\ Paulo Sérgio Moreira ${ }^{2}$ \\ Geraldo Lúcio de Faria ${ }^{2}$
}

\section{Resumo}

No presente trabalho, estudou-se a aplicação de modelos cinéticosde transformação martensíticadisponíveis na literatura técnica a fim de modelar curvas de fração da fase austenita transformada em martensita para o aço USISAR 80T, estrutural, microligado, de boa temperabilidade e considerado de boa soldabilidade. Ensaios dilatométricos foram realizados com taxa de aquecimento de $3^{\circ} \mathrm{C} / \mathrm{s}$ até a temperatura de austenitização de $920^{\circ} \mathrm{C}$, tempo de encharque de 180 segundos e taxas de resfriamento de $25^{\circ} \mathrm{C} / \mathrm{s}, 50^{\circ} \mathrm{C} / \mathrm{s}, 75^{\circ} \mathrm{C} / \mathrm{s}$ e $100^{\circ} \mathrm{C} / \mathrm{s}$. Um dos modelos estudados apresentou boa correlação com os dados experimentais para todas as taxas de resfriamento estudadas, sendo possível então a obtenção de constantes características da transformação estudada e a proposição de um modelo de previsibilidade para o aço USISAR 80T.

Palavras-chave: Cinética; Martensita; Modelamento; USISAR 80T.

\section{STUDY OF THE KINETICS OF MARTENSITIC TRANSFORMATION IN THE USISAR 80T MICROALLOYED STEEL}

\begin{abstract}
In this work, martensitic kinetic models, available in the technical literature, were studied aiming to model and to predict the austenite to martensite transformation for the USISAR 80T steel, which is a structural, microalloyed, with high hardenability and considered a great weldable steel. Dilatometrictests were performed with a heating rate of $3^{\circ} \mathrm{C} / \mathrm{s}$ until $920^{\circ} \mathrm{C}$, with a soaking time of 180 seconds and cooling rates of $25^{\circ} \mathrm{C} / \mathrm{s}, 50^{\circ} \mathrm{C} / \mathrm{s}, 75^{\circ} \mathrm{C} / \mathrm{s}$ and $100^{\circ} \mathrm{C} / \mathrm{s}$. One of the studied models showed good agreement with the experimental data for all the employed cooling rates, and it was possible to determinetransformation characteristic constants and suggest a previsibility model for the USISAR 80T steel. Keywords: Kinetics; Martensite; Modeling; USISAR 80T.
\end{abstract}

\section{INTRODUÇÃo}

A forma atérmica da transformação martensítica, presente nos aços carbono e baixa liga, é caracterizada por um aumento da fração da fase austenita transformada em martensita com o aumento do superresfriamento.Sabe-se que a composição química da fase austenita, o tamanho de grão austeníticoe a taxa de resfriamento, são fatores que exercem grande influência sobre a temperatura de início de transformação martensítica [I]. Já a taxa de transformação martensítica é influenciada pela taxa de resfriamento [2], composição química e fração de martensita já transformada [3].
Como os contornos de grãos austeníticos prévios não representam locais preferenciais para a nucleação de novos cristais de martensita, o tamanho do grão austenítico não influencia de forma significativa o desenvolvimento da transformação [4].

Diversos modelos foram propostos para descrever a fração de martensita formada a partir da fase austenita em função da temperatura durante o resfriamento contínuo, sendo - mais famoso deles o proposto por Koistinen-Marburger (1959) e que é descrito pela Equação I [3].

\footnotetext{
'Mestrando em Engenharia de Materiais - REDEMAT, Universidade Federal de Ouro Preto - UFOP, Ouro Preto, MG, Brasil. E-mail: samsouza2007@gmail.com ${ }^{2}$ Laboratório de Tratamentos Térmicos e Microscopia Óptica, Departamento de Engenharia Metalúrgica e de Materiais, Universidade Federal de Ouro Preto - UFOP, Ouro Preto, MG, Brasil

${ }^{3}$ Departamento de Engenharia Metalúrgica e de Materiais, Universidade Federal de Ouro Preto - UFOP, Ouro Preto, MG, Brasil
} 


$$
f=1-\exp \left(\alpha_{m}\left(M_{S}-T\right)\right.
$$

Onde $f$ representa a fração da fase austenita transformada em martensita, $\alpha_{m}$ é uma constante associada à taxa de transformação, $M_{S}$ é a temperatura de início detransformação martensítica e $T$ é a temperatura instantânea.

$\mathrm{O}$ aço estudado neste trabalho foi o USISAR 80T. Esse aço se enquadra no grupo dos aços de alta resistência e baixa liga, é considerado de alta temperabilidade e empregado no estado martensítico. Além disso, o USISAR 80T é considerado de boa soldabilidade e, portanto, é utilizado como soldado em muitas de suas aplicações [5].

É no contexto apresentado e com o objetivo de contribuir para o melhor entendimento da cinética de transformação martensítica do aço USISAR 80T e seu modelamento matemático, que o presente trabalho se propôs a estudar a aplicabilidade de alguns modelos cinéticos disponíveis na literatura especializada na descrição das curvas de fração da fase austenita transformada em martensita em função da temperatura. Acredita-se que os resultados obtidos no presente estudo são dados de base que poderão contribuir para o melhor entendimento da transformaçãomartensítica no aço USISAR 80T e, até mesmo, serem utilizados como dados de entrada em futuros trabalhos de simulação computacional de tensões residuais em juntas soldadas, ou produtos temperados.

\section{MATERIAIS E MÉTODOS}

\section{I Caracterização do Estado de Entrega}

Amostras representativas do aço USISAR 80T, em seu estado de entrega, foram submetidas a análises química e microestrutural. A análise química foi feita com o auxílio de três equipamentos: LECO-CS844 (determinação das concentrações de carbono e enxofre por absorção de infravermelho após fusão), LECO-TC436DR (determinação da concentração de nitrogênio por termocondutividade após fusão) e Thermo ARL-4460 (determinação da concentração dos demais elementos por espectrometria de emissão ótica).

As análises microestruturais foram realizadas em amostras devidamente polidas e atacadas com o reativo químico Nital $2 \%$ e conduzidas em um microscópio óptico de luz refletida, modelo DM 2700M, da fabricante Leica.

\subsection{Predição Termodinâmica das Fases Presentes no Equilíbrio}

Para o melhor entendimento da termodinâmica das transformações de fases do aço USISAR 80T, a sua composição química foi utilizada como dado de entrada de um algoritmo de simulação computacional para a avaliação da estabilidade das fases presentes em função da temperatura. A predição termodinâmica da estabilidade das fases e das frações em massa no equilíbrio foram realizadas por meio da utilização do software Matcalc, versão da base de dados mc_fe_v2.057.tdb [6].

\subsection{Ensaios Dilatométricos}

Para a realização dos estudos dilatométricos, corpos de prova cilíndricos e maciços foram preparados por usinagem, com dimensões de $3 \mathrm{~mm}$ de diâmetro por $10 \mathrm{~mm}$ de comprimento. Os ensaios foram realizados num dilatômetro de têmpera R.I.T.A L78 da fabricante Linseis. A pressão de vácuo utilizada foi da ordem de $7.10^{-2}$ bar e o gás de refrigeração foi o hélio analítico 5.0.

Inicialmente, corpos de prova foram submetidos a ensaios dilatométricos com o objetivo de se avaliar a taxa crítica de resfriamento abaixo da qual ocorre a formação de produtos difusionais. Amostras foram aquecidas a $3^{\circ} \mathrm{C} / \mathrm{s}$ até a temperatura de $920^{\circ} \mathrm{C}$, onde permaneceram por um tempo de 180 segundos para a completa austenitização. Em seguida, cada amostra foi resfriada com uma velocidade diferente. As taxas de resfriamento utilizadas foram de: $1{ }^{\circ} \mathrm{C} / \mathrm{s}, 3^{\circ} \mathrm{C} / \mathrm{s}$, $5^{\circ} \mathrm{C} / \mathrm{s}, 10^{\circ} \mathrm{C} / \mathrm{s}, 25^{\circ} \mathrm{C} / \mathrm{s}$ e $50^{\circ} \mathrm{C} / \mathrm{s}$. Nesta etapa, verificou-se que a taxa de resfriamento crítica é menor que $25^{\circ} \mathrm{C} / \mathrm{s}$.

Os ensaios dilatométricos executados com objetivo de se avaliar a cinética de transformação martensítica também foram conduzidos com temperatura de austenitização de $920^{\circ} \mathrm{C}$, taxa de aquecimento de $3^{\circ} \mathrm{C} / \mathrm{s}$ e tempo de encharque de 180 segundos. As taxas de resfriamento avaliadas foram: $25^{\circ} \mathrm{C} / \mathrm{s}, 50^{\circ} \mathrm{C} / \mathrm{s}, 75^{\circ} \mathrm{C} / \mathrm{s}$ e $100^{\circ} \mathrm{C} / \mathrm{s}$. Os dados dilatométricos obtidos foram tratados com o software Origin Pro 9.0. Curvas de fração de martensita transformada em função da temperatura foram obtidas por meio da aplicação da regra da alavanca às curvas de variação relativa dos comprimentos dos corpos de prova em função da temperatura [7].

\subsection{Modelamento Matemático}

O modelamento matemático das curvas de fração transformada em função da temperaturafoi feito com o auxílio do software Origin Pro 9.0. Além do modelo apresentado pela Equação I, os modelos matemáticos apresentados pelas Equações 2 [8] e 3 [9] também foram utilizados no modelamento, de forma a eleger aquele que apresenta melhor ajuste com os dados experimentais $[8,9]$.

$$
\begin{aligned}
& f=1-\exp \left(\beta \frac{\left(M_{S}-T\right)}{T}\right) \\
& f=1-\exp \left(\Omega\left(M_{S}-T\right)^{c}\right)
\end{aligned}
$$

Os parâmetros $\beta$ e $\Omega$ apresentados pelas Equações 2 e 3 , respectivamente, possuem significado semelhante àquele apresentado pelo parâmetro $\alpha_{m}$, apresentado pela Equação I . A Equação 3 é uma adaptação da Equação I por meio da proposição da constante $c$ como expoente da diferença de temperaturas. Os parâmetros $f, M_{s}$ e $T$, representam, em todas equações supracitadas, a fração da fase austenita 
transformada em martensita, a temperatura de início de transformação martensítica e a temperatura instantânea, respectivamente.

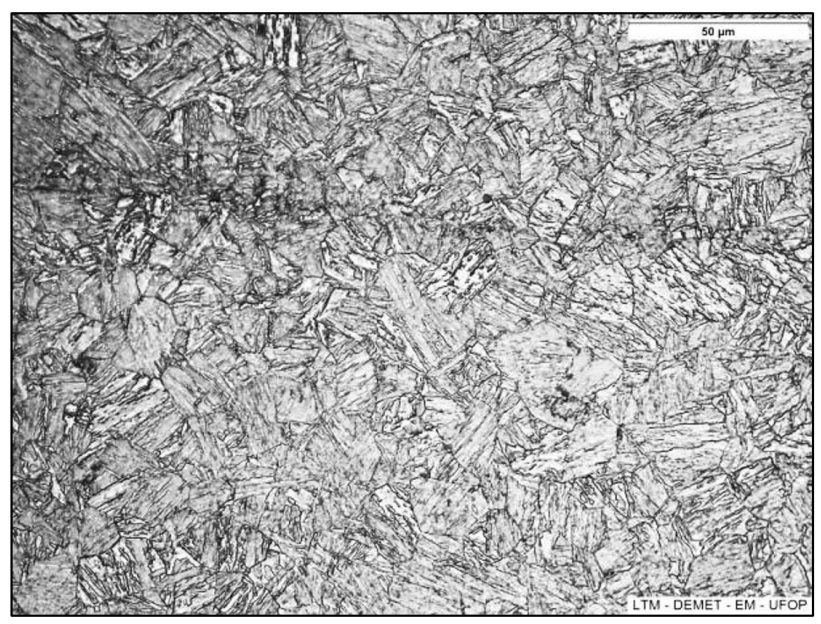

Figura I. Microestrutura do estado de entrega temperado e revenido do aço USISAR 80T. Ataque nital 2\%. 500x.

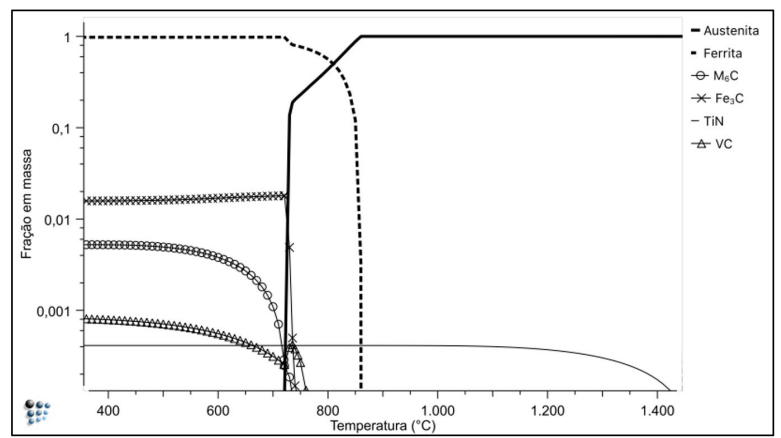

Figura 2. Estabilidade de fases em função da temperatura.

\section{RESULTADOS E DISCUSSÃO}

\section{I Caracterização do Estado de Entrega}

A Tabela I apresenta a composição química do aço em estudo. Observa-se a presença de elementos químicos gamagêneos, como o Mn e o C, que favorecem a temperabilidade sob o ponto de vista termodinâmico e cinético e de elementos químicos alfagêneos, como o $\mathrm{Cr}$, Mo e $\mathrm{Ti}$, que favorecem a temperabilidade apenas no aspecto de vista cinético. Destaca-se também a presença significativa de elementos bons formadores de carbonetos e nitretos, como o Mo, $\mathrm{Cr}$ e $\mathrm{Ti}$ [I0].

A Figura I ilustra a microestrutura do aço USISAR 80T no seu estado de entrega que é temperado e revenido.

A microestrutura é majoritariamente martensítica com morfologia aparente em ripas. Essa microestrutura está de acordo com o que era esperado para um aço baixo carbono, baixa liga, como o USISAR 80T quando temperado e revenido [10].

\subsection{Predição Termodinâmica das Fases Presentes no Equilíbrio}

A Figura 2 ilustra o cálculo das frações em massa das fases presentes no aço em função da temperatura considerando critérios de equilíbrio termodinâmico. De acordo com ela, os precipitados de $\mathrm{VC}, \mathrm{Fe}_{3} \mathrm{C}$ e $\mathrm{M}_{6} \mathrm{C}(\mathrm{M}=\mathrm{Mo}, \mathrm{Cr}$ ou $\mathrm{V})$ se solubilizariam em temperaturas inferiores a $920^{\circ} \mathrm{C}$, na qual o sistema será composto por austenita e precipitados de TiN.

\subsection{Velocidade Crítica de Resfriamento}

A Figura 3a ilustra curvas de variação relativa do comprimento dos corpos de prova em função da temperatura para as taxas de resfriamento de $50^{\circ} \mathrm{C} / \mathrm{s}, 25^{\circ} \mathrm{C} / \mathrm{s}, 10^{\circ} \mathrm{C} / \mathrm{s}, 5^{\circ} \mathrm{C} / \mathrm{s}$, $3^{\circ} \mathrm{C} / \mathrm{s}$ e $1{ }^{\circ} \mathrm{C} / \mathrm{s}$. Nela se observa que para taxas de resfriamento
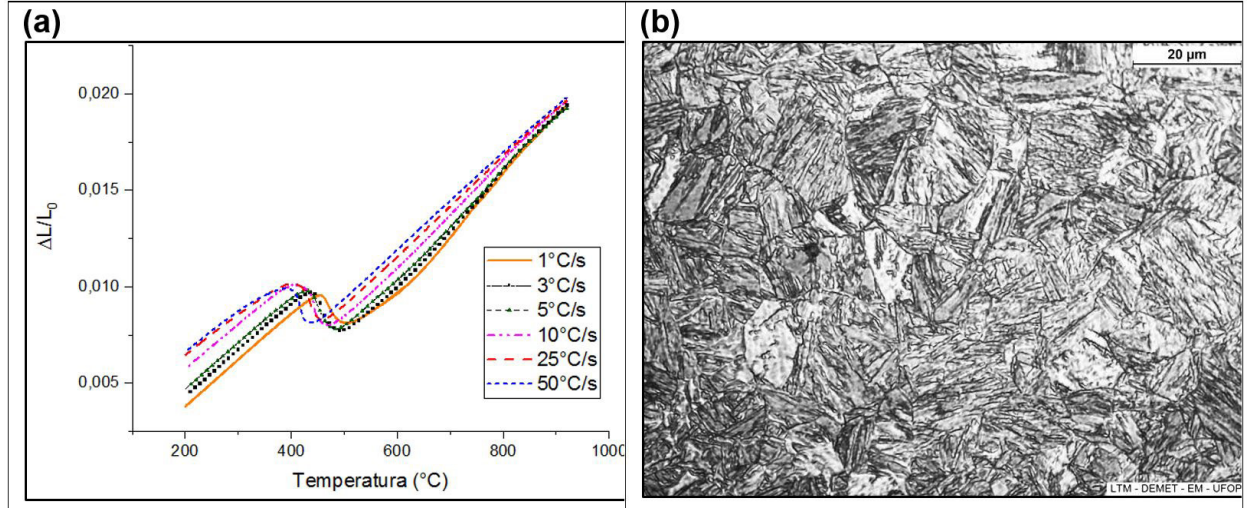

Figura 3. (a) Curvas de variação relativa dos comprimentos dos corpos de prova durante o resfriamento para várias taxas de resfriamento e (b) Microestrutura relativa a taxa de resfriamento de $25^{\circ} \mathrm{C} / \mathrm{s}$. Nital $2 \%$. MO. $500 \mathrm{x}$

Tabela I. Composição química do aço USISAR 80T (\% em peso)

\begin{tabular}{cccccccccc}
\hline $\mathbf{F e}$ & $\mathbf{C}$ & $\mathbf{S i}$ & $\mathbf{M n}$ & $\mathbf{P}$ & $\mathbf{S}$ & $\mathbf{C r}$ & $\mathbf{M o}$ & $\mathbf{T i}$ & $\mathbf{N}$ \\
\hline $\mathrm{Bal}$ & 0,1 & 0,3 & 0,9 & 0,02 & 0,003 & 0,7 & 0,5 & 0,02 & 0,005 \\
\hline
\end{tabular}


menores que $10^{\circ} \mathrm{C} / \mathrm{s}$, ocorre um desvio nas curvas dilatométricas para temperaturas acima de $550^{\circ} \mathrm{C}$, indicando a possibilidade de ocorrência de processos difusionais para as menores taxas de resfriamento. Portanto, pode-se presumir que para taxas de resfriamento maiores que $25^{\circ} \mathrm{C} / \mathrm{s}$ a austenita se transformará majoritariamente em martensita. A Figura $3 \mathrm{~b}$, que ilustra a microestrutura relativa à taxa de resfriamento de $25^{\circ} \mathrm{C} / \mathrm{s}$, que é martensítica, corrobora o resultado dilatométrico, ou seja, que a taxa crítica de resfriamento para esse aço na condição de austenitização empregada, abaixo da qual formam-se produtos difusionais, é menor que $25^{\circ} \mathrm{C} / \mathrm{s}$.

\subsection{Modelamento}

As Tabelas 2, 3 e 4 apresentam os valores dos parâmetros obtidos por meio dos ajustes não lineares das Equações I, 2 e 3, respectivamente, aos dados experimentais de fração transformada em função da temperatura.
Utilizando-se o parâmetro $R^{2}$ como referência, observa-se que a eficiência dos modelos apresentados para descrever a fração transformada em função da temperatura muda com a taxa de resfriamento. As Figuras 4a e 4b ilustramos ajustes dos modelos anteriormente apresentados aos dados experimentais para as taxas de resfriamento de $25^{\circ} \mathrm{C} / \mathrm{s}$ e $100^{\circ} \mathrm{C} / \mathrm{s}$, respectivamente.

O modelo apresentado pela Equação I, que foi proposto por Koistinen-Marburger, não apresentou boa correlação com os dados experimentais. Deve-se considerar que a racionalização do modelo empírico de Koistinen-Marburger considera que o volume médio dos cristais de martensita é constante e que a variação da diferença de energia livre de Gibbs entre as fases austenita e martensita com a temperatura é linear. Dessa forma, a constante $\alpha_{m}$ pode ser obtida a partir dos parâmetros supracitados, como ilustra a Equação (4) [II].

Tabela 2. Valores dos parâmetros do modelo apresentado pela Equação I

\begin{tabular}{cllc}
\hline Taxa & \multicolumn{1}{c}{$\boldsymbol{\alpha}_{\mathrm{m}}$} & \multicolumn{1}{c}{$\boldsymbol{M}_{\mathrm{s}}$} & $\boldsymbol{R}^{\mathbf{2}}$ \\
\hline 25 & $-0,0200 \pm 0,0004$ & $471,3 \pm 0,5$ & 0,896 \\
50 & $-0,0224 \pm 0,006$ & $446,4 \pm 0,6$ & 0,914 \\
75 & $-0,0314 \pm 0,0009$ & $438,0 \pm 0,4$ & 0,934 \\
100 & $-0,0411 \pm 0,0009$ & $431,5 \pm 0,3$ & 0,964 \\
\hline
\end{tabular}

Tabela 3. Valores dos parâmetros do modelo apresentado pela Equação 2

\begin{tabular}{cccc}
\hline Taxa & $\boldsymbol{\beta}$ & $\boldsymbol{M}_{s}$ & $\boldsymbol{R}^{2}$ \\
\hline 25 & $-8,34 \pm 0,15$ & $471,1 \pm 0,6$ & 0,923 \\
50 & $-9,0 \pm 0,2$ & $446,4 \pm 0,6$ & 0,934 \\
75 & $-12,8 \pm 0,4$ & $438,1 \pm 0,4$ & 0,941 \\
100 & $-16,7 \pm 0,4$ & $431,6 \pm 0,3$ & 0,963 \\
\hline
\end{tabular}

Tabela 4. Valores dos parâmetros apresentados pela Equação 3

\begin{tabular}{ccccc}
\hline Taxa & $\Omega$ & $\boldsymbol{M}_{s}$ & $\boldsymbol{c}$ & $\boldsymbol{R}^{\mathbf{2}}$ \\
\hline 25 & $-0,0018 \pm 0,0003$ & $464,7 \pm 0,5$ & $1,71 \pm 0,04$ & 0,997 \\
50 & $-0,006 \pm 0,001$ & $439,9 \pm 0,8$ & $1,46 \pm 0,05$ & 0,988 \\
75 & $-0,108 \pm 0,006$ & $428,8 \pm 0,2$ & $0,77 \pm 0,01$ & 0,988 \\
100 & $-0,11 \pm 0,007$ & $426,2 \pm 0,2$ & $0,77 \pm 0,02$ & 0,987 \\
\hline
\end{tabular}
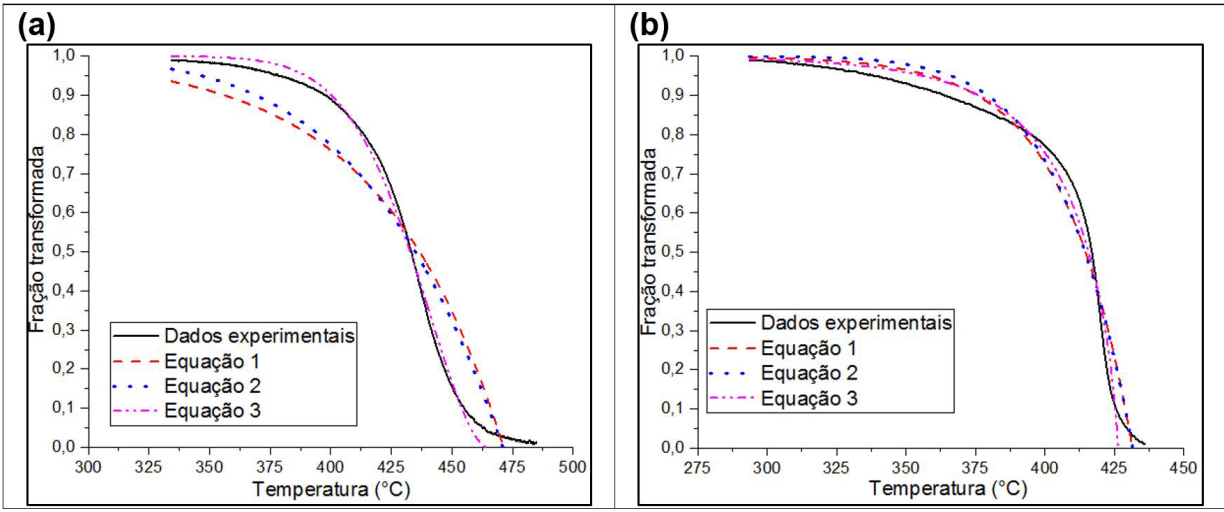

Figura 4. Ajustes dos modelos cinéticos apresentados pelas Equações I, 2 e 3 aos dados experimentais para todo o intervalo de transformação. (a) $25^{\circ} \mathrm{C} / \mathrm{s}$ e (b) $100^{\circ} \mathrm{C} / \mathrm{s}$. 


$$
\alpha_{m}=-\bar{V} \cdot C_{2} \cdot d\left(\Delta G^{\left(\gamma \rightarrow \alpha^{\prime}\right)}\right) / d T
$$

Onde, $\bar{V}$ representa o volume médio dos cristais de martensita, $C_{2}$ é uma constante de ajuste e $d\left(\Delta G^{\left(\gamma \rightarrow \alpha^{\prime}\right)}\right) / d T$ é a derivada em relação à temperatura da diferença de energia livre de Gibbs entre as fases austenita e martensita [I I].

Bhadeshiae Khan [12] calcularama derivada da diferença da energia livre de Gibbs entre as fases austenita e martensita em relação a temperatura para o aço $300 \mathrm{M}(\mathrm{Fe}-0,44 \% \mathrm{C}$ I ,74\%Si-0,67\%Mn- I,85\%Ni-0,83\%Cr0,39\%Mo-0,09\%V) contendo uma variedade de concentrações de carbono devido a transformações bainíticas prévias.Segundo os autores, os valores obtidos foram praticamente constantes nos intervalos de ocorrência da transformação martensítica. Nesse contexto, a consideração de que a derivada da diferença de energia livre de Gibbs entre as fases austenita e martensita é constante durante a transformação martensítica é razoável. Porém, segundo Phadham e Ansell [3], esse valor pode decrescer para baixas temperaturas.

No entanto, o modelo de Koistinen-Marburger não é eficiente em considerar alguns fatores: i) $O$ volume médio dos cristais de martensita não é necessariamente constante ao longo de toda a transformação. McMurtrie e Magee et al. [12] mostraram experimentalmente que para as ligas $\mathrm{Fe}-23,8 \% \mathrm{Ni}-0,42 \% \mathrm{C}$ (em peso) e $\mathrm{Fe}-28,5 \% \mathrm{Ni}-0,4 \%$ C, o volume médio dos cristais de martensita é praticamente independente da fração transformada até frações da ordem de 0,55 . Contudo, para frações transformadas maiores o efeito de particionamento tende a ser relevante, e o volume médio dos cristais de martensita tende a diminuir [13]. ii) Desconsideração do aumento da resistência a deformação da austenita remanescente com o aumento da fração transformada, fenômeno que ocorre porque a transformação martensítica é acompanhada de uma expansão volumétrica que deve ser acomodada pela austenita ainda não transformada por meio de uma deformação plástica/elástica, aumentando assim a sua resistência a uma deformação posterior e, por consequência,também o superresfriamento necessário para que a transformação prossiga [3]. iii) Desconsideração do fenômeno denominado autocatálise, no qual a evolução da fração transformada é acompanhada da criação de defeitos que atuam como locais propícios à nucleação de novos cristais martensíticos [13]. iv) O modelo de Koistinen-Marburger assume que todos os locais que possuem as características de núcleos martensíticos possuem a mesma energia de ativação, no entanto, esses defeitos não são idênticos e são ativados em diferentes graus de superresfriamento abaixo de $M_{S}[13]$.

A desconsideração desses fatores faz com que o modelo apresentado por Koistinen-Marburger apresente curva em " $C$ ", diferentemente dos dados experimentais que apresentam curvas em "S" [I3]. É importante, todavia, ressaltar que os efeitos de autocatálise e de aumento da resistência da austenita remanescente à deformação produzem resultados antagônicos sobre a taxa de transformação martensítica. A taxa de transformação aumenta com o aumento da fração transformada devido à ocorrência da autocatálise até que o efeito relativo ao aumento da resistência da austenita remanescente à deformação se torne preponderante, desse ponto em diante a taxa de transformação martensítica começa a diminuir com o avançar da transformação martensítica [3].

Observa-se ainda um aumento da correlação do modelo de Koistinen-Marburger com os dados experimentais com o aumento da taxa de resfriamento. Isso acontece porque o aumento da taxa de resfriamento é acompanhado de um aumento da derivada da diferença de energia livre de Gibbs entre as fases austenita e martensita com a temperatura, que tem por consequência um aumento na taxa de nucleação martensítica [2]. Como a velocidade de crescimento dos cristais de martensita nos aços é comparável à velocidade do som, ou seja, independente do tempo [14], a taxa de transformação martensítica também aumenta e por consequência as curvas de fração da fase austenita transformada em martensítica passam a apresentar um formato mais próximo de " $\mathrm{C}$ ".

O modelo apresentado pela Equação 2 apresentou comportamento semelhante ao modelo apresentado pela Equação I. Ele, assim como o modelo apresentado pela Equação I, considera que o volume médio dos cristais de martensita é constante durante toda a transformação e que a variação da diferença de energia livre de Gibbs com a temperatura entre as fases austenita e martensita é linear [8]. Apesar de a sua dedução teórica considerar o efeito da autocatálise na transformação [8], diferentemente do modelo apresentado pela Equação I, isso não é traduzido em seu comportamento. Além disso, assim como o modelo de Koistinen-Marburger ele não é eficiente em considerar que os locais propícios à nucleação de novos cristais de martensita possuem diferentes energias de ativação.

Já o modelo apresentado pela Equação 3, empírico, possui boa concordância com os dados experimentais para todas as taxas de resfriamento estudadas e isso se deve ao fato de ele representar curvas em " $\mathrm{S}$ ", assim como as curvas experimentais de fração da fase austenita transformada em martensita em função da temperatura.

De forma a estudar a possibilidade de modelamento da cinética de transformação martensítica em função da taxa de resfriamento, os parâmetros do modelo apresentado pela Equação 3, que apresentou melhor correlação com os dados experimentais, foram apresentados em função da taxa de resfriamento. As Figuras 5a e 5 b ilustram como os parâmetros $\Omega$ e $c$, respectivamente, variam com a taxa de resfriamento.

Nos dois casos estudados os valores do parâmetro $\Omega$ e $c$ diminuíram com o aumento da taxa de resfriamento no intervalo entre $25^{\circ} \mathrm{C} / \mathrm{s}$ e $75^{\circ} \mathrm{C} / \mathrm{s}$. No entanto, quando os valores relativos a taxa de resfriamento de $75^{\circ} \mathrm{C} / \mathrm{s}$ são comparados àqueles relativos a taxa de resfriamento de $100^{\circ} \mathrm{C} / \mathrm{s}$, não se verifica mudança significativa devida ao efeito da taxa de resfriamento. Isso acontece porque o efeito da taxa de resfriamento sobre o formato das curvas de fração 

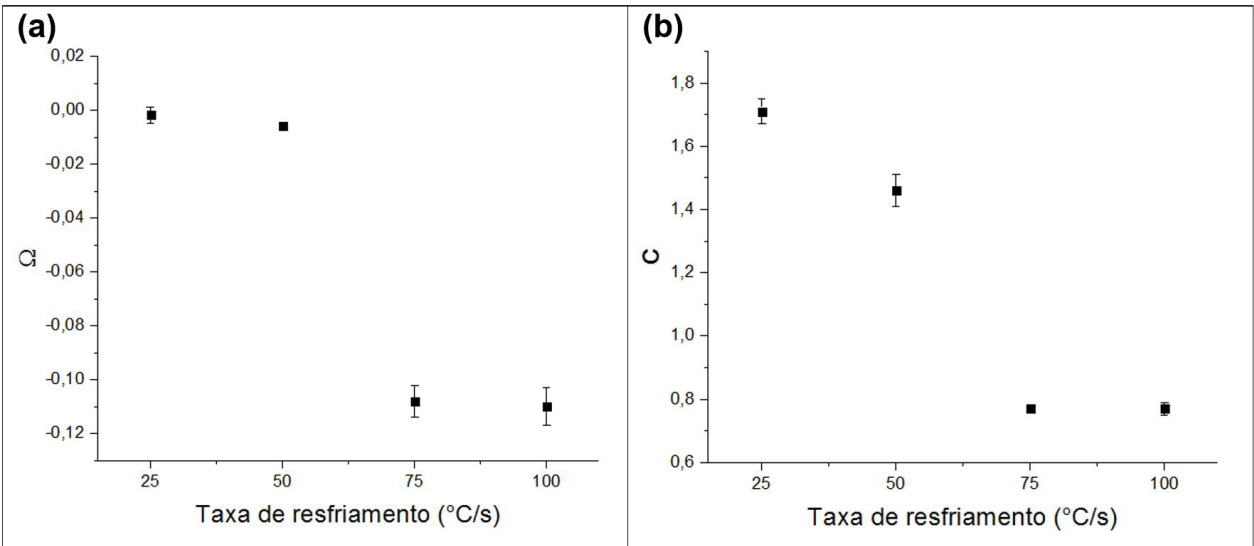

Figura 5. Correlação entre os parâmetros $\Omega$ (a) e $c$ (b), da Equação 3, com a taxa de resfriamento.

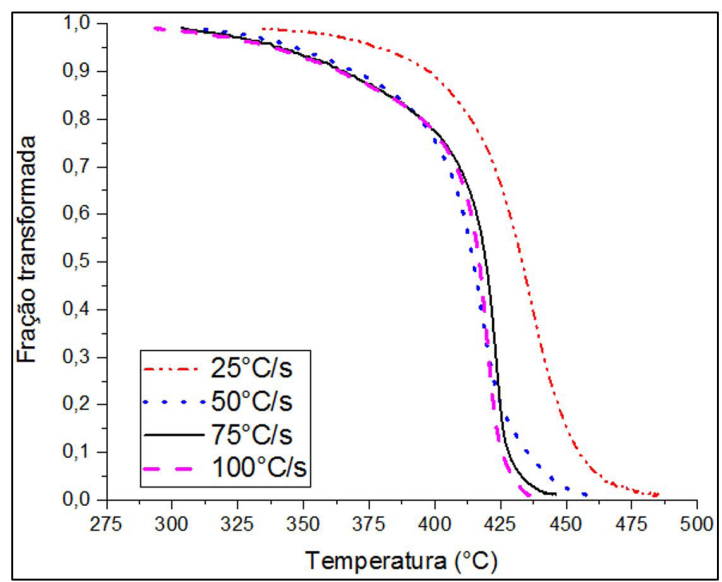

Figura 6. Influência da taxa de resfriamento no formato das curvas de fração da fase austenita transformada em martensita.

da fase austenita transformada em martensita em função da temperatura se tornou menos pronunciado. A Figura 6 ilustra o efeito da taxa de resfriamento sobre o formato das curvas de fração da fase austenita transformada em martensita em função da temperatura.

\section{CONCLUSÕES}

Foi observado que para o aço microligado USISAR 80T, com microestrutura inicial martensítica, quando austenitizado a $920^{\circ} \mathrm{C} \mathrm{com} \mathrm{taxa} \mathrm{de} \mathrm{aquecimento} \mathrm{de} 3^{\circ} \mathrm{C} / \mathrm{s}$ e tempo de encharque de 180 segundos, a taxa crítica de resfriamento, abaixo da qual ocorre a formação de produtos difusionais, é menor que $25^{\circ} \mathrm{C} / \mathrm{s}$.

Análises termodinâmicas conduzidas como auxílio do software Matcalc,permitiram concluir que a composição da fase austenita é praticamente invariável no intervalo entre $900^{\circ} \mathrm{Ce}$ $1300^{\circ} \mathrm{C}$, estando os elementos $\mathrm{Cr}$, Mo e V em solução sólida.

Os modelos cinéticos disponíveis na literatura encontram dificuldade em descrever as curvas de fração da fase austenita transformada em martensita e isso se deve a dificuldade de explicar e de modelar os fenômenos que acompanham a transformação, como a influência da fração da fase austenita já transformada no prosseguimento da transformação. No entanto, um dos modelos estudados apresentou razoável correlação com os dados experimentais, permitindo que os parâmetros relativos a ele fossem correlacionados com as taxas de resfriamento utilizadas nos experimentos. Nesse contexto, dados a cerca da transformação martensítica no aço USISAR 80T foram produzidos e disponibilizados. Essas informações podem ser utilizadas com confiabilidade para a previsão da evolução da transformação martensítica no supracitado aço e até mesmo para a realização de simulações computacionais.

\section{Agradecimentos}

Os autores agradecem ao CNPq pela concessão de bolsa de Mestrado (I33285/2016-2) e pela bolsa de produtividade em pesquisa (303426/2017-9).

\section{REFERÊNCIAS}

I Gao Q, Wang C, Qu F, Wang Y, Qiao Z. Martensite transformation kinetics in 9Cr-1.7W-0,4Mo-Co ferritic steel. Journal of Alloys and Compounds. 2014;610:322-330. 
2 Gao Q, Liu Y, Di X, Yu L, Yan Z. Martensite transformation in the modified high Cr ferritic heat-resistant steel during continuous cooling. Journal of Materials Research. 20I 2;27(2I):2779-2789.

3 Phadham R, Ansell G. Kinetics of the martensite transformation in athermal Fe-C-Ni-Cr alloys. Metallurgical Transactions. A, Physical Metallurgy and Materials Science. 1978;9A(6):793-80I.

4 Bohemen SMC, Sietsma J. Kinetics of martensite formation in plain carbon steels: critical assessment of possible influence of austenite grain boundaries and autocatalysis. Materials Science and Technology. 20 I4;30(9): I024- 033.

5 Usinas Siderurgicas de Minas Gerais S.A. - USIMINAS. Catálogo online. [acesso em 14 fev. 20 I7]. Disponível em: http://www.usiminas.com/solucoes/wp-content/uploads/sites/5/2013/I2/su-catalogo-online.pdf

6 Kozeschnik E, Buchmayr B. MatCalc - A simulation tool for multicomponent thermodynamics, diffusion and phase transformation kinetics. In autor(es). Mathematical modelling of weld phenomena. London: CRC Press; 200I. p. 349-361.

7 Motycka P, Köver M. Evaluation methods of dilatometer curves of phase transformation. São Caetano do Sul: COMAT; 2012.

8 Guimarães J, Rios P. Modeling lath martensite transformation curve. Metallurgical and Materials Transactions. A, Physical Metallurgy and Materials Science. 20I3;44A(I):2-4.

9 Lee S, Tyne C. A kinetics model for martensite transformation in plain carbon and low-alloyed steels. Metallurgical and Materials Transactions. A, Physical Metallurgy and Materials Science. 20I2;43A(2):422-427.

10 Krauss G. Steels: processing, structure and performance. Materials Park: ASM International; 2005.

II Bohemen S, Siestma J, Hermans M, Richardson I. Kinetics of the martensitic transformation in low-alloy steel studied by means of acoustic emission. Acta Materialia. 2003;5I(I4):4I83-4I96.

12 McMurtrie DG, Magge CL. The average volume of martensite plates during transformation. Metallurgical Transactions. 1970;1:3185-3319.

13 Khan S, Bhadeshia $\mathrm{H}$. Kinetics of martensitic transformation in partially bainitic 300M steel. Materials Science and Engineering A. 1990; 129(2):257-272.

14 Porter D, Easterling K, Sherif M. Diffusionless transformation. In: Porter D, Easterling K, Sherif M. Phase transformations in metals and alloys. Boca Raton: CRC Press; 2009. p. 383-435.

Recebido em: 19 Jul. 2018

Aceito em: I 2 Set. 2019 